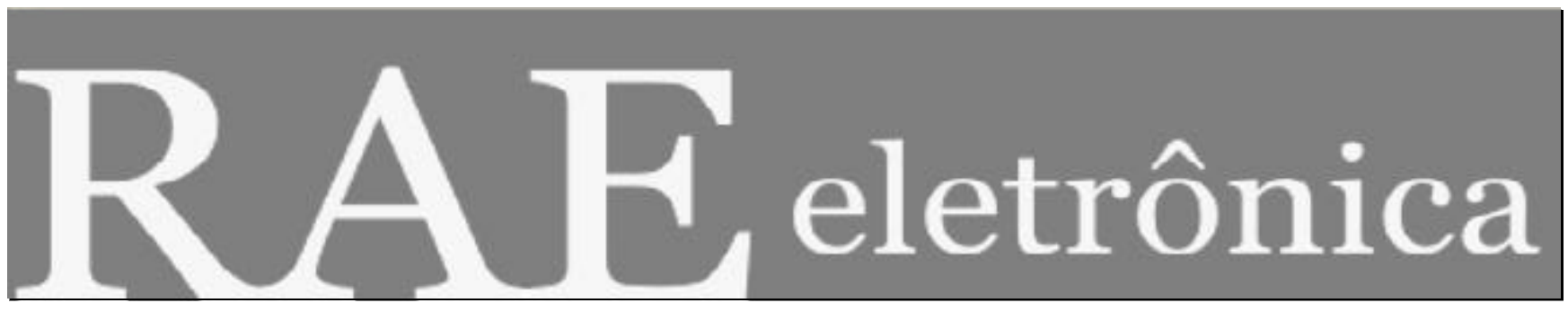

\title{
SURPRESA E A FORMAÇÃO DA SATISFAÇÃO DO CONSUMIDOR
}

Por:

Juliano A. Larán, University of Florida.

Carlos Alberto Vargas Rossi, UFRGS.

RAE-eletrônica, v. 5, n. 1, Art. 1, jan./jun. 2006

http://www.rae.com.br/eletronica/index.cfm?FuseAction=Artigo\&ID=2016\&Secao=ARTIGOS\&Volu $\mathrm{me}=5 \&$ Numero $=1 \& \mathrm{Ano}=2006$

CCopyright, 2006, RAE-eletrônica. Todos os direitos, inclusive de tradução, são reservados. É permitido citar parte de artigos sem autorização prévia desde que seja identificada a fonte. A reprodução total de artigos é proibida. Os artigos só devem ser usados para uso pessoal e nãocomercial. Em caso de dúvidas, consulte a redação: raeredacao@fgvsp.br.

A RAE-eletrônica é a revista on-line da FGV-EAESP, totalmente aberta e criada com o objetivo de agilizar a veiculação de trabalhos inéditos. Lançada em janeiro de 2002, com perfil acadêmico, é dedicada a professores, pesquisadores e estudantes. Para mais informações consulte o site www.rae.com.br/eletronica.

RAE-eletrônica

ISSN 1676-5648

(C2006 Fundação Getulio Vargas - Escola de Administração

de Empresas de São Paulo.

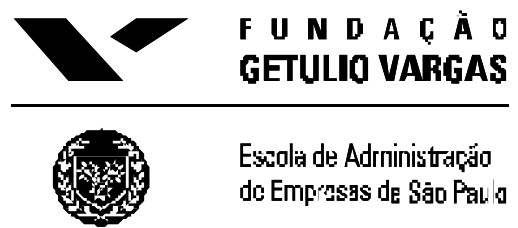




\title{
RESUMO
}

O artigo apresenta o teste de um novo modelo, teoricamente lastreado, da influência das surpresas positiva e negativa no processo de formação da satisfação. Os resultados de uma pesquisa survey demonstram uma influência negativa da surpresa negativa na satisfação, totalmente mediada pelo afeto negativo, e uma influência positiva da surpresa positiva na satisfação, totalmente mediada pelo afeto positivo. $\mathrm{O}$ artigo contribui para o entendimento das principais emoções que determinam a satisfação, com foco na surpresa, que pode ter valência tanto positiva quanto negativa.

\section{PALAVRAS-CHAVE}

Marketing, comportamento do consumidor, satisfação, emoções, surpresa.

\begin{abstract}
The article proposes a new theoretical model based on the influence of both, negative and positive surprise, upon the consumer satisfaction formation process. An empirical survey found a negative influence of negative surprise, mediated by negative effect, and a positive influence of positive surprise on satisfaction, also mediated by positive effect. Discussion of the results and implications for further research are presented.
\end{abstract}

\section{KEYWORDS}

Marketing, consumer behavior, satisfaction, emotions, surprise. 


\section{INTRODUÇÃO}

As emoções ${ }^{1}$ têm um papel fundamental na decisão do consumidor. Desde o final da década de 1980 estudos têm descrito a dimensionalidade e a influência das emoções na satisfação ou insatisfação do consumidor (Westbrook, 1987; Westbrook e Oliver, 1991; Oliver, 1993; Mano e Oliver, 1993). Entretanto, em comparação com o processamento de informações e a pesquisa sobre processo de decisão, sabe-se muito menos sobre o papel das emoções no comportamento do consumidor (Bagozzi et al., 1999), mesmo que essa abordagem já venha sendo reclamada há algum tempo na disciplina (Holbrook e Hirschman, 1982). Em modelo proposto por Bagozzi et al. (1999), a surpresa é considerada um fator de influência em todo o processo emocional de formação da satisfação. De acordo com Vanhamme e Snelders (2001), a surpresa pode aumentar ou diminuir a satisfação, pois ela pode ser tanto negativa quanto positiva. Para Oliver (1989), a ocorrência da surpresa parece amplificar os sentimentos antecedentes da satisfação e a própria satisfação, mas seu papel ainda não está claro. Não obstante, esta reação afetiva parece atuar de forma significativa sobre a satisfação e o encantamento do consumidor (Westbrook e Oliver, 1991; Oliver et al., 1997; Vanhamme, 2002c) e na busca de relacionamentos com os clientes (Vanhamme e Lindgreen, 2001).

Considerando esta lacuna na literatura de marketing, este artigo objetiva verificar o papel da surpresa no processo emocio nal de formação da satisfação. A principal contribuição das análises feitas aqui é na continuidade das pesquisas sobre as emoções dos consumidores, como a surpresa as influencia e como estas variáveis estão relacionadas com a satisfação. Com este propósito foi construído um modelo teórico, fundado na literatura sobre o tema, que inclui surpresas positiva e negativa, afetos positivo e negativo. O modelo estrutural foi testado em uma survey com uma amostra de estudantes, e seus principais resultados e conclusões são apresentados na seqüência do artigo.

\section{SURPRESA DO CONSUMIDOR}

Juntamente com a satisfação, a retenção, a lealdade e a insatisfação do consumidor, o tema "surpresa" integra os comportamentos pós-compra do consumidor. Assim como no contexto acadêmico internacional, também no Brasil a satisfação do consumidor tem atraído significativamente os pesquisadores (Rossi e Slongo, 1997; Farias e Santos, 1998; Urdan e Rodrigues, 1998; Marchetti e

Prado, 2001). Também têm sido objeto de pesquisas no Brasil a retenção e a lealdade (Rosa e Kamakura, 2002) e a insatisfação (Beber, 2000; Santos, 2001; Giglio e Chauvel, 2002). 


\section{SURPRES A E A FORMAÇÃO DA SATISFAÇÃO DO CONSUMIDOR}

Juliano A. Larán - Carlos Alberto Vargas Rossi

Em relação ao tema "surpresa", apesar de ser alvo de estudos em psicologia, ainda há um número reduzido de publicações que o aborde em comportamento do consumidor (são exceções os trabalhos de Vanhamme, 2000, 2001, 2002c; Vanhamme e Lindgreen, 2001). Muitos pesquisadores têm incitado ao estudo da surpresa (Westbrook e Oliver, 1991; Derbaix e Pham, 1991) e apontam a ausência de um conjunto teórico sobre esse assunto na literatura de marketing (Oliver et al., 1997).

Tangenciando esse tema, são encontradas na literatura três abordagens conceituais acerca dos eventos inesperados, causadores ou antecedentes da surpresa. A primeira é o conceito da desconfirmação de expectativas (Stiensmeier-Pelster et al., 1995) e afirma que o evento surpreendente desconfirma as crenças implícitas, de acordo com as quais o ocorrido era improvável em determinada situação. A segunda abordagem é o conceito da divergência do schema teórico (Meyer et al., 1991;

Rumelhart, 1984) e afirma que a surpresa será produzida por um evento que diverge do schema; de acordo com Rumelhart (1984), um schema é um tipo privado, normalmente informal, desarticulado de teoria sobre a natureza de objetos, eventos ou situações. E a terceira abordagem é o conceito de modelo neural (Sokolov, 1963 apud Vanhamme, 2002a), que representa um sistema de células no qual é estocada a informação relativa a um estímulo que já se apresentou várias vezes; em outras palavras, o sistema nervoso produz um modelo exato de propriedades dos objetos exteriores que agem sobre os órgãos sensitivos. Para essa abordagem, uma reação de surpresa ocorre quando os estímulos produzidos não correspondem ao modelo neural que foi construído nas apresentações anteriores.

Mas nem todo evento inesperado é considerado surpreendente, e a teoria da norma se torna essencial no entendimento desse aparente paradoxo. Ela postula que o indivíduo cria padrões de comparação após a experiência, e esses padrões podem ser usados para avaliar quão surpreendente foi o evento (Kahneman e Miller, 1986). Esse mecanismo é chamado de "processamento retrocedente", porque o próprio evento influencia os padrões que serão utilizados para julgar seu grau de surpresa ou (a)normalidade.

Por ter sido pouco pesquisada, também existem dúvidas se a surpresa se caracteriza como uma emoção. Portanto, antes de passar às hipóteses de pesquisa que norteiam o trabalho, a questão a seguir será abordada.

\section{A surpresa é uma emoção?}

Assim como no caso dos comportamentos pós-compra, nota-se que há um interesse na academia brasileira pelo tema "emoções" (Arruda, 2001; Rossi e Hor-Meyll, 2001; Laran, 2002), que acompanha a rota de pesquisa internacional (por exemplo, Hirschmann e Holbrook, 1982; Frijda, 1986; Ortony et 
al., 1988; Bagozzi et al., 1999). Não existe ainda um consenso a respeito de a surpresa ser ou não uma emoção. Boa parte da diversidade conceitual em torno do tema reside nas diferentes definições de emoções. Por exemplo, Ortony et al. (1988) consideram que as emoções devem ter uma valência positiva ou negativa - de modo que a surpresa não seria uma emoção, por ser neutra (os eventos podem gerar tanto surpresa positiva quanto negativa). Essa visão é compartilhada por Kumar (1996), para quem a surpresa é considerada uma "não-emoção" capaz de intensificar e também modificar o tipo de emoções sentidas por um indivíduo. Para outros autores, a surpresa é uma emoção, mesmo que não seja considerada necessariamente uma emoção básica (Oately e Johnson-Laird, 1987).

Izard (1977) afirma que a surpresa não é uma emoção no mesmo sentido que a alegria ou a tristeza, pois não tem as mesmas características de outras emoções. Segundo Izard, a surpresa é ativada por um aumento drástico na ativação neural. Percebe-se, entretanto, que Izard, autor da escala de emoções utilizada neste estudo, também trata a surpresa como uma emoção, mesmo que diferenciada. $\mathrm{Na}$ abordagem discreta das emoções, ela pode ser considerada uma emoção primária, de base ou prototípica (Plutchik, 1980) ou fundamental (Izard e Buechler, 1980).

Segundo Vanhamme (2002a), os pesquisadores que trabalham atualmente a emoção surpresa especialmente um grupo de trabalho da Universidade de Bielenfeld, na Alemanha -, a descrevem como uma síndrome de reações, como uma entidade teórica que se caracteriza por suas manifestações observáveis em três níveis: a) nível comportamental: expressões faciais distintas, cessação das atividades, concentração da atenção sobre o que desperta a surpresa; b) nível fisiológico: mudança no ritmo dos batimentos do coração e da respiração, melhor condutibilidade da pele; c) nível subjetivo: experiência subjetiva de surpresa, verbalizações espontâneas das experiências subjetivas.

Dessa forma, a surpresa pode ser considerada uma emoção? Para fins deste artigo será considerada afirmativamente, com características próprias em relação às outras emoções, que serão aprofundadas no modelo testado neste artigo.

\section{RELAÇÃO ENTRE SURPRESA E SATISFAÇÃO}

Para Kumar (1996), a surpresa tem uma importante função de ampliação da memória ao focar a atenção em seu evento causador. Esse foco de atenção leva à melhor memorização do evento surpreendente. 
A surpresa também pode exercer a função de amplificadora de emoções subseqüentes (Vanhamme, 2002b), como sugerem a maioria dos estudos de marketing sobre processos emocionais de formação da satisfação (Oliver, 1989; Westbrook e Oliver, 1991; Oliver e Westbrook, 1993) e do encantamento (Oliver et al., 1997; Kumar et al., 2001). Vanhamme (2002a) afirma que devido à ativação intrínseca à surpresa, ela é capaz de ativar não apenas as emoções positivas e negativas subseqüentes, mas também a própria satisfação, visto que ela possui um componente afetivo (Westbrook, 1980) e está relacionada ao grau de ativação (Oliver et al., 1997).

Para Oliver e Westbrook (1993), a desconfirmação de expectativas está implícita na surpresa, e por isso ela pode estar relacionada a subseqüentes sentimentos positivos e negativos. Neste estudo, os autores verificaram que consumidores com níveis maiores de satisfação e insatisfação apresentaram os maiores níveis de surpresa. Dessa forma, sugere-se que a surpresa tem uma valência neutra, podendo adquirir uma tonalidade positiva ou negativa de acordo com as emoções geradas a partir do evento surpreendente.

Com relação à sua dimensionalidade, Oliver e Westbrook (1993) encontraram a emoção surpresa da escala DES (Differential Emotions Scale), de Izard (1977), como localizada em uma dimensão independente das outras emoções. Em outro estudo, Westbrook e Oliver (1991) investigaram a relação entre os construtos emoção e satisfação para identificar padrões de respostas emocionais a experiências com produtos. Os resultados foram semelhantes em relação à dimensionalidade da emoção surpresa. Com base nestes estudos, afeto positivo, afeto negativo e surpresa foram estudados em três dimensões diferentes na presente pesquisa.

Westbrook e Oliver (1991) comprovaram que a surpresa pode estar relacionada tanto a emoções positivas (surpresa agradável) quanto negativas (surpresa desagradável). Como antecedentes de altos níveis de satisfação, foram encontrados pelos autores sentimentos de felicidade/contentamento e encantamento. Os mesmos autores, em outros dois artigos (Oliver, 1989; Oliver e Westbrook, 1993), afirmam que a surpresa parece atuar como um "amplificador" da avaliação de experiências de consumo positivas e negativas, em julgamentos de satisfação. Essas relações foram encontradas em experimento realizado por Vanha mme (2002-b), o que leva às duas primeiras hipóteses de pesquisa:

- $\quad \mathrm{H}_{1}$ : a surpresa positiva terá influência positiva no afeto positivo e influência negativa no afeto negativo;

- $\quad \mathrm{H}_{2}$ : a surpresa negativa terá influência negativa no afeto positivo e influência positiva no afeto negativo. 
Em 1993, Oliver analisou a satisfação e a insatisfação como agentes causais de sentimentos positivos e negativos, respectivamente. Com esse estudo, Oliver demonstrou a complexidade do processo de formação da satisfação, com resultados que sugerem o mecanismo pelo qual as respostas afetivas medeiam os efeitos dos atributos na satisfação, de forma independente da desconfirmação, que também foi testada na pesquisa.

Mano e Oliver (1993) encontraram que duas dimensões de avaliação do produto - performance utilitária e hedônica - são antecedentes causais de duas dimensões de afeto - prazer e ativação - e da satisfação com o produto. Como em estudos anteriores (Westbrook, 1987), dimensões de afetos positivo e negativo foram encontradas como antecedentes de estados de satisfação e insatisfação, respectivamente.

Vanhamme e Snelders (2001) observam que esses estudos não oferecem suporte empírico definitivo à influência da surpresa na satisfação, e que deve haver mediadores entre as duas variáveis. Os autores acreditam que a alta ativação inerente à surpresa amplificaria as emoções subseqüentes em relação ao produto ou serviço (tal como a alegria) e que essas emoções aumentariam o nível de satisfação do consumidor. Ao testar empiricamente essa idéia, Vanhamme (2002b) obteve resultados que comprovam a mediação da alegria no que tange ao relacionamento da surpresa com a satisfação. $O$ modelo teórico testado na presente pesquisa foi originado nessas diferentes rotas de construção de teoria sobre a surpresa e os temas relacionados. Inclui tanto emoções positivas quanto negativas, possibilitando o teste das seguintes hipóteses:

- $\quad \mathrm{H}_{3}$ : o efeito da surpresa positiva (negativa) na satisfação será positivo (negativo) e totalmente mediado pelos construtos do modelo;

- $\quad \mathrm{H}_{4}$ : o afeto positivo (negativo) terá influência positiva (negativa) na satisfação.

A Figura 1 apresenta o modelo estrutural completo de formação da satisfação, indicando a direção das hipóteses de pesquisa. 


\section{Figura 1 - Modelo estrutural de formação da satisfação}

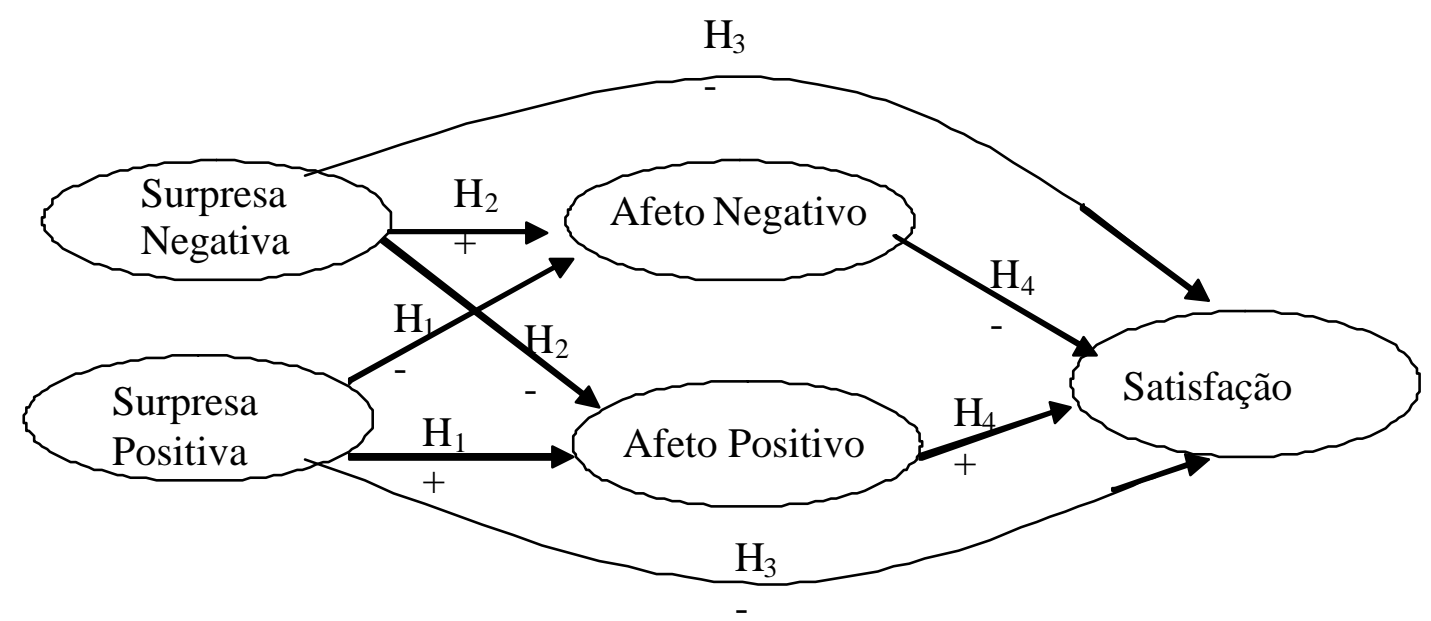

\section{MÉTODO}

O método de pesquisa incluiu duas etapas, uma exploratória e uma descritiva, e uma série de decisões a respeito de amostragem, de produtos a serem avaliados e de tipos de medidas utilizadas, para que não fosse comprometido o rigor necessário ao estudo. A seguir, apresentam-se as etapas da pesquisa e as decisões que serviram de base para a consecução dos objetivos.

\section{Etapa exploratória}

Essa etapa teve como objetivos desenvolver o modelo a ser testado empiricamente; definir teoricamente as possíveis emoções do consumidor frente a experiências de consumo com produtos de diferentes níveis de envolvimento; definir o melhor design de pesquisa; identificar, na literatura, as escalas a serem utilizadas no estudo; e construir o instrumento de coleta de dados.

Para que esses objetivos fossem atingidos, foram desenvolvidas as seguintes atividades: a) revisão da literatura, que propiciou o desenvolvimento das hipóteses de pesquisa, a identificação das melhores escalas para a medição do construto e a definição do melhor design; b) entrevistas em profundidade (12 indivíduos, alunos de cursos de graduação de três universidades brasileiras), a fim de entender o papel das surpresas positiva e negativa na formação de satisfação e de identificar eventuais 
emoções de consumo alternativas àquelas encontradas nas escalas (não foram encontradas emoções alternativas às das escalas); e, c) construção do instrumento de coleta de dados para a etapa descritiva.

\section{Etapa descritiva}

Sobre a etapa descritiva serão apresentados os aspectos concernentes aos respondentes, aos produtos pesquisados, ao questionário, às medidas utilizadas e aos procedimentos estatísticos de análise dos dados.

\section{Respondentes}

A população da pesquisa foi formada pelos cursos de graduação de três universidades brasileiras. A amostra, selecionada por conveniência, abrangeu um total de 447 estudantes que participaram de uma pesquisa survey que pedia que fizessem parte de uma pesquisa sobre avaliações, sentimentos e satisfação de consumidores em relação a alguns produtos.

\section{Produtos}

Foram distribuídos questionários com opções de produtos de alto e baixo envolvimento, a fim de contemplar um eventual papel moderador do envolvimento do consumidor entre a surpresa e a satisfação. O questionário de alto envolvimento apresentava as seguintes opções de produto: computador pessoal, carro, roupa, viagem de turismo e telefone celular. O questionário de baixo envolvimento apresentava as seguintes opções: CD, jornal, shampoo e filme para máquina fotográfica. Os questionários foram divididos de forma equivalente entre produtos de alto e baixo envolvimento, para que as experiências não ficassem restritas a tipos específicos de produtos. Essa abordagem possibilitou maior generalização dos resultados, pois o modelo foi avaliado para experiências de consumo com produtos de diversos níveis de envolvimento. Essa variável foi medida com o Novo Perfil de Envolvimento (NIP - New Involvement Profile) de Jain e Srinivasan (1990), com um envolvimento médio de 4,44 (escala de 1 a 7), com as médias variando entre os limites de 1,47 e 6,47. Essa escala é particularmente adequada ao uso no mercado brasileiro, tendo em vista estudos anteriores que verificaram sua aplicabilidade no Brasil (Fonseca, 1999; Fonseca e Rossi, 1999; Fonseca e Rossi, 2000).

O preço médio dos produtos analisados é de $\mathrm{R} \$ 2.906,00$ (d.p. = R \$ 6.815,00), e os produtos tinham sido adquiridos em média há 1 ano (d.p. = 1 ano e 5 meses). Os elevados desvios-padrão desses 
valores indicam a variedade de produtos analisados, com várias faixas de preço e experiências analisadas em diversos períodos. Cabe salientar que estava sendo analisada toda a experiência do respondente com o produto, de modo que o tempo de compra, por vezes elevado, não int erferisse no preenchimento do questionário.

\section{Procedimentos de coleta}

Foram realizadas duas etapas de pré-teste com indivíduos da população estudada, para verificação da correção e do entendimento do questionário. Alterações derivadas da aplicação do pré-teste serão comentadas na seqüência.

$\mathrm{Na}$ etapa descritiva os questionários foram auto-administrados. Os estudantes universitários preencheram os questionários nas salas de aula, seguindo orientações dadas pelos próprios pesquisadores. A primeira parte do questionário visava suscitar um produto cuja experiência de consumo deveria ser avaliada. Quatro tipos de questionário foram aplicados em igual número, pois além de alto e baixo envolvimento, também foram avaliadas experiências geradoras de emoções e surpresas positivas e negativas. Por exemplo, para o grupo de alto envolvimento e surpresa negativa, os respondentes foram instruídos a lembrar, dentre as opções de produtos apresentadas, uma das compras mais complexas que já tinham feito, para a qual tivessem gastado um tempo considerável pensando antes de fazer a compra e pudessem lembrar de forma precisa seu desempenho e uso. O produto poderia ter sido pago por outra pessoa, desde que o respondente tivesse sido o responsável pela decisão de compra. Por fim, pedia-se que fosse escolhido o produto que lhe havia trazido o maior número de emoções e surpresas negativas.

Após a lembrança de um produto específico que cada um pudesse avaliar, foram feitas perguntas sobre a intensidade das emoções despertadas pelo produto, quão surpresos (positiva e negativamente) eles ficaram e sua satisfação com a experiência de consumo.

\section{Medidas}

As medidas utilizadas na pesquisa provêm da literatura de emoções, da psicologia e da satisfação do consumidor. A escolha das medidas se baseou na sua utilização em estudos anteriores e pela comprovação de sua validade e confiabilidade.

As emoções de consumo foram medidas com a Escala de Emoções Diferenciais (DES Differential Emotions Scale) de Izard (1977), que passou por tradução reversa realizada por três 
acadêmicos de marketing com fluência em inglês e português. A escala de Izard é constituída de sete emoções negativas (raiva, desgosto, desprezo, angústia, medo, vergonha e culpa) e duas emoções positivas (interesse e alegria). A intensidade das emoções foi medida em escala ancorada por 1 - muito fraco e 5 - muito forte. Inicialmente, todas as emoções da escala eram medidas por três itens, mas após os pré-testes alguns itens foram retirados por serem redundantes ou de difícil compreensão.

Vanhamme (2000) mediu a surpresa de formas diferentes, como expressões faciais, estimulação neural e relatos verbais, não havendo evidência de que uma exerça a função de mensuração melhor que as outras. Dessa forma, no presente estudo, a surpresa, a exemplo das emoções, foi medida com os itens da DES (Izard, 1977), que apresenta três itens para a medida de surpresa (surpreso(a), admirado(a), perplexo(a)). Para testar o papel da surpresa negativa e positiva, os itens de medida foram sucedidos das palavras positivamente ou negativamente, formando três itens para cada valência específica da surpresa. A intensidade da surpresa foi medida em escala ancorada por 1 - muito fraco e 5 - muito forte.

A satisfação foi medida com seis dos 12 itens da escala de Oliver (1997), em escala ancorada por 1 - discordo plenamente e 7 - concordo plenamente. A diminuição do número de itens se justifica pelo fato de que, segundo Bruner II e Hensel (1998), a escala mantém validade interna mesmo com menos do que seus 12 itens originais. A seleção dos itens, bem como do número de itens a serem utilizados, foi realizada pelos autores com base em estudos anteriores sobre antecedentes emocionais da satisfação.

\section{Procedimentos estatísticos de análise dos dados}

O método de análise do modelo teórico seguiu sugestão de Hair et al. (1998), para quem o principal objetivo da modelagem de equações estruturais é explicar uma série de relacionamentos de dependência entre construtos latentes (inter-relacionados simultaneamente). Dessa forma, o uso dessa técnica estatística é adequado aos objetivos de pesquisa, quais sejam, avaliar relações entre variáveis latentes, medidas por variáveis observáveis.

Para tanto, foram realizados os seguintes procedimentos: análise inicial da base de dados brutos, incluindo análises caso-a-caso e assuntos relacionados à distribuição e relacionamentos entre as variáveis; análise das estatísticas descritivas dos construtos; análises fatoriais confirmatórias dos construtos surpresa positiva, surpresa negativa, afeto positivo, afeto negativo e satisfação; análises da validade discriminante e da multicolinearidade; análise fatorial confirmatória do modelo de 
mensuração completo; estimação do modelo estrutural e teste dos modelos rivais. Para a análise dos dados foram utilizados os pacotes estatísticos SPSS $10.0^{\circledR}$ e AMOS $4^{\circledR}$.

\section{RESULTADOS}

Os resultados da pesquisa serão apresentados na seguinte ordem: análise inicial da base de dados, análise dos respondentes, análise das diferenças entre os produtos investigados, o modelo de mensuração e o modelo estrutural.

\section{Análise inicial da base de dados}

Duas grandes categorias de análises de dados brutos foram utilizadas: análises caso-a-caso, como valores omissos (ou dados ausentes) e outliers (ou valores extremos); e assuntos relacionados à distribuição e relacionamentos entre as variáveis, como normalidade, linearidade e homoscedasticidade.

Quanto aos valores omissos, a análise do banco de dados mostrou que 1,6\% foi o percentual máximo de valores omissos entre as variáveis (segundo Kline [1998] esta magnitude não deve ser maior do que 5 a 10\% das observações para cada variável) e que todos os valores eram aleatórios, o que permitiu a correção do problema sem a necessidade de eliminação de variáveis. Foi utilizado o método de substituição dos dados omissos pela média, conforme sugerido por Hair et al. (1998).

Os outliers multivariados foram identificados por meio do exame da Distância Mahalanobis (D2), que indica a distância multivariada entre os valores de um caso individual e as médias amostrais. $\mathrm{Na}$ análise, assumindo $\mathrm{p}<0,001$, foram identificados 63 indivíduos outliers. Após a comparação dos índices de ajustamento do modelo entre a base de dados sem os outliers e a base completa, verificou-se que todos os índices eram superiores com a base completa, o que levou à opção de não eliminar nenhum outlier. Também se verificou a normalidade, a linearidade e a homoscedasticidade dos dados.

O cálculo dos valores de assimetria e curtose e da medida de Mardia, de curtose multivariada, registrou a ocorrência de não-normalidade em algumas das variáveis manifestas do modelo, principalmente no que diz respeito à curtose negativa. Utilizou-se, então, um método gráfico de avaliação, seguindo Tabachnick e Fidell (2001). Todas as variáveis intervalares mostraram algum tipo de assimetria, sendo os casos mais sérios os das variáveis de afeto negativo, com assimetria à esquerda e curtose negativa, pois a maioria das respostas concentrou-se no valor 1 da escala. Procurou-se 
transformar estas variáveis não-normais, levando em conta que o principal problema encontrado foi o de curtose negativa. As fórmulas de transformação sugeridas por Kline (1998), como a raiz cúbica das respostas, foram aplicadas. No entanto, após a checagem dos resultados da transformação, não foi identificada melhoria nos ajustamentos do modelo nem na confiabilidade das escalas, o que levou à utilização, na análise final, dos valores originais das variáveis.

Gráficos também foram utilizados na avaliação da linearidade, com o uso de scatterplots das variáveis para a identificação de qualquer padrão não-linear nos dados (Hair et al., 1998). Essa análise indicou relações lineares apenas entre as variáveis dos eixos X e Y. Já a multicolinearidade, resultante do mau condicionamento dos dados (Jagpal, 1982) e caracterizada pela existência de correlações de 0,90 ou mais entre as variáveis, não foi apontada no banco de dados da pesquisa.

\section{Respondentes}

Os entrevistados foram selecionados em três universidades brasileiras, por conveniência, entre diferentes cursos de graduação. Um total de 447 estudantes participou de uma survey. A amostra constituiu-se de 52,4\% de respondentes do sexo masculino e 47,6\% do sexo feminino, com idades entre 18 e 48 anos (média = 24 anos, d.p. = 5,4). Quanto à renda, 16,6\% dos entrevistados têm renda familiar mensal de até $\mathrm{R} \$ 1.000,00,25,9 \%$ de $\mathrm{R} \$ 1.001,00$ a $\mathrm{R} \$ 2.000,00,22,9 \%$ de $\mathrm{R} \$ 2.001,00$ a $\mathrm{R} \$ 3.000,00$, $15,9 \%$ têm renda entre $\mathrm{R} \$ 3.001,00$ e $\mathrm{R} \$ 4.000,00$ e 12,5\% dos respondentes têm renda familiar acima de $\mathrm{R} \$ 5.000,00$.

\section{Difere nças entre os produtos investigados}

Os 447 questionários foram assim distribuídos da seguinte forma:

- 232 questionários referentes a produtos de alto envolvimento, sendo 110 na combinação Alto Envolvimento - Surpresa Positiva e 122 na combinação Alto Envolvimento - Surpresa Negativa;

- 216 questionários referentes a produtos de baixo envolvimento, sendo 99 na combinação Alto Envolvimento - Surpresa Positiva e 117 na combinação Alto Envolvimento - Surpresa Negativa.

Foi analisada a variância das respostas da variável envolvimento, tendo sido realizado um teste ANOVA para avaliar as diferenças entre os resultados destes quatro tipos de questionário. Foram encontradas diferenças significativas entre os questionários de alto e baixo envolvimento. Ainda assim, as médias de envolvimento nos quatro tipos de combinações foram de moderadas a altas (todas acima 
de 4, lembrando que a escala era de 1 a 7). Essa baixa discriminação no envolvimento com os produtos levou à decisão de analisar os dados referentes à amostra, no restante da pesquisa, como um todo, ou seja, considerando o total de 447 questionários.

\section{Modelo de mensuração}

O modelo de mensuração foi testado por meio de análise fatorial confirmatória. A análise do modelo abrangeu a confiabilidade das medidas, a variância extraída de cada construto, a significância das cargas dos indicadores nos fatores, a validade discriminante entre os construtos e os índices de ajustamento. A confiabilidade dos fatores, analisada pela medida de confiabilidade composta e pelo alpha de Cronbach, foi satisfatória, já que apenas a medida de afeto positivo ficou um pouco abaixo dos padrões no que concerne ao alpha de Cronbach (abaixo de 0,70), mas acima dos padrões de confiabilidade composta (acima de 0,50, de acordo com Hair et al., 1998). Da mesma forma, a medida de variância extraída, que deve ser superior a 0,50, é satisfatória, apesar de a surpresa positiva e do afeto positivo terem ficado em níveis um pouco abaixo para esta variável. As cargas dos indicadores em seus respectivos fatores foram todas significativas ao nível de 0,05 e os itens de um mesmo fator apresentaram correlação entre si, indicando validade convergente. Esses dados encontram-se em detalhe na Tabela 1.

Tabela 1 - Médias, desvios-padrão e medidas de confiabilidade das escalas

\begin{tabular}{l|l|l|l|l}
\hline Construto & Média & $\begin{array}{l}\text { Coeficiente } \\
\text { Alpha }\end{array}$ & $\begin{array}{l}\text { Confiabilidade } \\
\text { Composta }\end{array}$ & $\begin{array}{l}\text { Variância } \\
\text { Extraída }\end{array}$ \\
\hline Surpresa positiva & $\mathbf{2 , 9 7}$ & $\mathbf{0 , 7 3}$ & $\mathbf{0 , 7 5}$ & $\mathbf{0 , 5 0}$ \\
\hline Surpresa negativa & $\mathbf{2 , 2 3}^{1}$ & $\mathbf{0 , 8 2}$ & $\mathbf{0 , 8 3}$ & $\mathbf{0 , 6 2}$ \\
\hline Afeto Positivo & $\mathbf{3 , 3 4}^{1}$ & $\mathbf{0 , 5 9}$ & $\mathbf{0 , 8 2}$ & $\mathbf{0 , 6 1}$ \\
\hline Afeto Negativo & $\mathbf{2 , 0 0}$ & $\mathbf{0 , 9 0}$ & $\mathbf{0 , 9 0}$ & $\mathbf{0 , 5 6}$ \\
\hline Satisfação & $\mathbf{5 , 0 3}^{1}$ & $\mathbf{0 , 9 1}$ & $\mathbf{0 , 9 1}$ & $\mathbf{0 , 6 2}$ \\
\hline
\end{tabular}

Fonte: dados da pesquisa

(1) escala de 1 a 5

(2) escala de 1 a 7

A validade discriminante dos construtos do modelo também foi avaliada. De acordo com Bagozzi e Phillips (1982), a validade discriminante entre dois construtos pode ser avaliada restringindo-se a apenas um o parâmetro que estabelece a relação entre os construtos. Compara-se esse modelo com outro em que o parâmetro não é fixado e se faz um teste de diferença qui-quadrado. Se o 
teste indicar uma diferença qui-quadrado significante quando a correlação não é restrita, isso indica haver validade discriminante entre os construtos. Esse procedimento foi realizado entre cada construto e todos os outros, e todos os testes indicaram haver validade discriminante entre os construtos constituintes do modelo. Por fim, também com o objetivo de avaliar a validade discriminante, comparoutse a variância extraída de cada construto e as suas variâncias compartilhadas, ou seja, o quadrado das correlações entre os construtos. Esse teste é sugerido por Fornell e Larcker (1981) e, segundo Spreng et al. (1996), é um teste mais robusto para análise da validade discriminante. Os resultados não corroboraram a validade discriminante entre os construtos surpresa positiva e afeto positivo e entre os construtos surpresa negativa e afeto negativo. Cabe ressaltar que essa correlação entre os construtos acima já era esperada, visto que a surpresa, de acordo com sua valência, dá uma tonalidade às emoções subseqüentes, de modo que é normal que estes construtos tenham correlação.

Como última análise, o modelo de mensuração mostrou índices de ajustamento razoáveis diante da natureza ainda exploratória do estudo, com qui-quadrado $\left.\chi^{2}\right)=633,81$ (p < 0,001); GL = 197; $\chi^{2} / \mathrm{GL}=3,22 ; \mathrm{GFI}=0,88 ; \mathrm{AGFI}=0,85 ; \mathrm{IFI}=0,93 ; \mathrm{TLI}=0,92 ; \mathrm{CFI}=0,93 ; \mathrm{RMSEA}=0,07$. Portanto, o modelo de mensuração parece refletir cada construto conceitual e teoricamente, com bons índices de ajustamento, confiabilidade e validades convergente e discriminante. Com isso, a próxima etapa é analisar o modelo estrutural e suas inter-relações.

\section{Modelo estrutural}

A análise está focada no modelo estrutural apresentado na Figura 1. As hipóteses foram testadas por meio dos índices de ajustamento do modelo e das magnitudes e níveis de significância dos parâmetros (Kline, 1998). O modelo estrutural foi estimado com o uso do critério de máxima verossimilhança. O modelo hipotetizado inicialmente mostrou bons índices de ajustamento, com qui-quadrado $=774,61$ $(\mathrm{p}<0,001) ; \mathrm{GL}=202 ; \chi^{2} / \mathrm{GL}=3,83 ; \mathrm{GFI}=0,87 ; \mathrm{AGFI}=0,83 ; \mathrm{IFI}=0,91 ; \mathrm{TLI}=0,90 ; \mathrm{CFI}=0,91$; RMSEA $=0,08$. Como o objetivo da pesquisa é testar um novo modelo teórico, uma abordagem de modelos rivais foi utilizada para amplificar a discussão sobre a adequação do modelo inicialmente proposto pelos autores.

Modelo Rival 1 - testando a influência direta da surpresa positiva na satisfação: como não se sabe exatamente o papel da surpresa positiva no processo de formação da satisfação, foi testado se seu efeito na satisfação é completamente mediado pelos construtos do modelo. Ao comentar essa relação, Vanhamme (2003) afirmou ter encontrado resultados diversos em diferentes estudos, havendo ou não a 
relação de mediação aqui analisada. Esse modelo alternativo teve um qui-quadrado de 774,12 (p<.001); $201 \mathrm{GL} ; \chi^{2} / \mathrm{GL}=3,85 ; \mathrm{GFI}=0,87$; AGFI = 0,83; IFI =0,91; TLI = 0,90; CFI =0,91; $\mathrm{RMSEA}=0,08$. Percebe-se não ter havido uma melhora nos índices de ajustamento como um todo, e um teste de diferença qui-quadrado foi conduzido, cujo resultado não indicou mudança significativa do modelo (variação no qui-quadrado de 0,49). Da mesma forma, foi analisado o caminho entre surpresa positiva e satisfação, que tampouco foi significante (coeficiente padronizado de $-0,42$, estatística $t=-0,75, \mathrm{p}=$

0,45). O efeito indireto da surpresa positiva na satisfação é 0,46 (valor padronizado), significativo a $\mathrm{p}=0,00$. Isso indica que a surpresa positiva tem um forte efeito na satisfação, que é completamente mediado pelos construtos do modelo, oferecendo suporte para a $\mathrm{H}_{3}$ de pesquisa.

Modelo Rival 2 - testando a influência direta da surpresa negativa na satisfação: o efeito direto da surpresa negativa na satisfação, não totalmente mediado pelos construtos do modelo, foi igualmente testado em um modelo rival. Vanhamme (2003) afirma que, a exemplo dos estudos suscitando surpresa positiva, nos estudos com surpresa negativa também encontrou resultados em que o efeito da surpresa era mediado pelos construtos do modelo e outros em que havia uma relação direta. Portanto, esse é um modelo que merece análise. O modelo rival 2 teve um qui-quadrado de 774,12 (p<.001); 201 GL; $\chi^{2} / \mathrm{GL}=3,85 ; \mathrm{GFI}=0,87 ; \mathrm{AGFI}=0,83 ; \mathrm{IFI}=0,91 ; \mathrm{TLI}=0,90 ; \mathrm{CFI}=0,91 ; \mathrm{RMSEA}=0,08$. Os índices são os mesmos daqueles encontrados no teste de mediação da surpresa positiva, com diferença qui-quadrado não significante e um novo caminho que se comportou da mesma forma (coeficiente padronizado de $-0,20$, estatística $\mathrm{t}=-0,75)$. Além disso, o efeito indireto da surpresa negativa na satisfação é -0,54 (valor padronizado), significativo a p =0,00. Isso indica haver completa mediação do efeito da surpresa negativa na satisfação pelos construtos do modelo, oferecendo novo suporte para a $\mathrm{H}_{3}$ de pesquisa.

No geral, a abordagem de modelos rivais comprova resultados anteriores (Oliver, 1989; Oliver e Westbrook, 1993), em que a surpresa funciona como um amplificador, pois influencia emoções, que por sua vez influenciarão a satisfação. Considerando a estratégia de modelos rivais, o modelo estrutural inicialmente proposto foi adotado como o modelo final estimado das relações entre os construtos. O R para a satisfação no modelo final foi de 0,51, sugerindo que os construtos emocionais explicam uma considerável quantidade de variância da satisfação. A Tabela 2 apresenta os coeficientes padronizados e seus níveis de significância. 
SURPRES A E A FORMAÇÃO DA SATISFAÇÃO DO CONSUMIDOR

Juliano A. Larán - Carlos Alberto Vargas Rossi

Tabela 2 - Parâmetros estimados do modelo estrutural

\begin{tabular}{l|l|l|l}
\hline Caminho Estrutural & $\begin{array}{l}\text { Coeficiente } \\
\text { Padronizado }\end{array}$ & $\mathbf{p}$ & Estatística t \\
\hline Surpresa Positiva $\rightarrow$ Afeto Negativo $\left(\mathbf{H}_{1}\right)$ & $-\mathbf{0 , 0 9}$ & $\mathbf{0 , 0 0}$ & $-\mathbf{2 , 8 1}$ \\
\hline Surpresa Positiva $\rightarrow$ Afeto Positivo $\left(\mathbf{H}_{\mathbf{1}}\right)$ & $\mathbf{0 , 9 0}$ & $\mathbf{0 , 0 0}$ & $\mathbf{1 2 , 5 5}$ \\
\hline $\begin{array}{l}\text { Surpresa Negativa } \rightarrow \text { Afeto Negativo } \\
\left(\mathbf{H}_{2}\right)\end{array}$ & $\mathbf{0 , 9 5}$ & $\mathbf{0 , 0 0}$ & $\mathbf{1 7 , 0 4}$ \\
\hline Surpresa Negativa $\rightarrow$ Afeto Positivo $\left(\mathbf{H}_{2}\right)$ & $-\mathbf{0 , 4 3}$ & $\mathbf{0 , 0 0}$ & $-\mathbf{- 1 1 , 0 6}$ \\
\hline Surpresa positiva $\rightarrow$ Satisfação $\left(\mathbf{H}_{3}\right)$ & $-\mathbf{0 , 4 2}$ & $\mathbf{0 , 4 5}$ & $-\mathbf{0 , 7 5}$ \\
\hline Surpresa negativa $\rightarrow$ Satisfação $\left(\mathbf{H}_{3}\right)$ & $\mathbf{- 0 , 2 0}$ & $\mathbf{0 , 4 5}$ & $-\mathbf{0 , 7 5}$ \\
\hline Afeto Positivo $\rightarrow$ Satisfação $\left(\mathbf{H}_{4}\right)$ & $\mathbf{0 , 4 7}$ & $\mathbf{0 , 0 0}$ & $\mathbf{9 , 2 0}$ \\
\hline Afeto Negativo $\rightarrow$ Satisfação $\left(\mathbf{H}_{4}\right)$ & $-\mathbf{0 , 3 6}$ & $\mathbf{0 , 0 0}$ & $-\mathbf{7 , 1 7}$ \\
\hline
\end{tabular}

A análise dos parâmetros do modelo (veja a Tabela 2) mostra que todas as relações hipotetizadas tiveram valores significantes, na direção prevista, sustentando todas as hipóteses propostas inicialmente. Sustentando a $\mathrm{H}_{1}$, a surpresa positiva tem influência negativa no afeto negativo (caminho padronizado $=-0,09, t=-2,81$ ) e influência positiva no afeto positivo (caminho padronizado $=0,90, t=12,55)$. Dando sustentação à $\mathrm{H}_{2}$, percebe-se que a surpresa negativa tem influência contrária e mais forte no afeto negativo (caminho padronizado $=0,95, t=17,04$ ) e no afeto positivo (caminho padronizado $=-0,95, t=-11,06)$. No que se refere à $\mathrm{H}_{4}$, também sustentada pelos dados, o afeto negativo tem influência negativa na satisfação (caminho padronizado $=-0,36, t=-7,17$ ) mais fraca do que a influência positiva do afeto positivo (caminho padronizado $=0,47, t=9,20$ ), o que confirma a hipótese de pesquisa mas deixa margem a discussões sobre o impacto das emoções na satisfação. Esses resultados legitimam o modelo de influência da surpresa no processo emocional de formação da satisfação.

\section{DISCUSSÃO E CONCLUSÕES}

A proposta desta pesquisa foi contribuir com os estudos sobre o papel da surpresa no processo de formação da satisfação. Além disso, questões metodológicas estão incluídas entre os achados da pesquisa, atendendo ao chamado de Vanhamme (2000) para que fossem aprimorados os métodos de medição da surpresa. O modelo teórico teve sua concepção inicial baseada nos estudos de Vanhamme (2002a), que tratava a influência da surpresa na satisfação a partir de várias abordagens metodológicas. Entretanto, seus estudos jamais consideraram que os consumidores poderiam ter surpresas positivas e negativas na mesma experiência de consumo, bem como emoções positivas e negativas. Além disso, a 
avaliação de experiências com diversos produtos, variados em seus níveis de envolvimento, agrega substância ao conteúdo de pesquisas anteriores, que utilizaram um produto específico em cada uma delas.

Conforme os dados da Tabela 2, pode-se dizer que a surpresa positiva de fato gera emoções - de caráter positivo - e que essas emoções têm uma influência positiva forte na satisfação. Ou seja, uma experiência de consumo em que haja surpresa positiva tem influência na satisfação de forma independente, além daquela dos modelos cognitivos tradicionais (como, por exemplo, desconfirmação de expectativas). Além da geração de emoções positivas, a surpresa terá influência negativa sobre possíveis emoções negativas relativas à experiência de consumo, ou seja, a ocorrência de uma situação surpreendente, positivamente, irá inibir a ocorrência de emoções negativas, o que também irá impactar na satisfação. Além disso, o efeito indireto da surpresa positiva na satisfação foi significativo, fornecendo suporte ao papel da surpresa positiva como um amplificador das emoções positivas, que irão subseqüentemente influenciar a satisfação. A surpresa negativa tem um impacto semelhante ao da surpresa positiva, mas no sentido contrário. Seu papel, conforme evidenciado pelos dados, é de amplificar emoções negativas que irão ter um impacto negativo na satisfação, provavelmente resultando em insatisfação. Quanto aos testes de mediação, o efeito indireto da surpresa negativa na satisfação foi significativo, fornecendo suporte ao papel da surpresa negativa como um amplificador das emoções negativas, que irão impactar a satisfação.

Um dado importante é a força maior da surpresa negativa em comparação com a surpresa positiva, facilmente visualizada nos dados da Tabela 2. O padrão de respostas também pode ser visualizado nos efeitos indiretos da surpresa na satisfação, que é mais impactada indiretamente pelo afeto negativo. O "poder" da surpresa negativa no modelo parece encontrar explicação na prospect theory, de Kahneman e Tversky (1979), que afirmam que perdas têm impacto maior que ganhos. Ao atribuir pesos a este impacto mais forte das perdas do que dos ganhos, Tversky e Kahneman (1992) estimaram que a razão perdas/ganhos é de 2,5, algo não confirmado na mesma magnitude, mas na mesma direção dos resultados desta pesquisa.

Também é importante ressaltar a confirmação de estudos anteriores no que diz respeito à importância de variáveis emocionais na formação da satisfação. Os construtos utilizados no modelo aqui proposto, todos de cunho emocional, explicaram sozinhos mais de $50 \%$ da variância da satisfação, o que atesta a capacidade preditiva do modelo e oferece caminhos para que determinantes afetivos e cognitivos sejam utilizados na busca de uma explicação ainda mais significativa. 
Em suma, os resultados mostraram que uma experiência de surpresa positiva torna os consumidores significantemente mais satisfeitos, enquanto uma experiência de surpresa negativa irá diminuir a satisfação consideravelmente, sempre havendo a mediação dos afetos correspondentes. O aspecto dual da surpresa provou estar agindo nas experiências de consumo descritas pelos consumidores, pois sentimentos positivos e negativos parecem estar misturados em muitas dessas experiências. Esses resultados são convergentes com aqueles de Williams e Aaker (2002), que encontraram uma propensão a experimentar e aceitar emoções ambivalentes em indivíduos nãoamericanos.

\section{Implicações acadêmicas e sugestões de futuras pesquisas}

Essa pesquisa é a primeira tentativa de testar um novo modelo. Apesar de terem sido replicados alguns resultados de pesquisas anteriores (Vanhamme 2002a, 2002b), são recomendadas futuras investigações dos inter-relacionamentos dos construtos presentes no modelo. A ocorrência de surpresas positivas e negativas durante um período maior de tempo é um caminho interessante para futuras pesquisas, pois a satisfação e as emoções variam com o tempo e ambas as valências da surpresa podem ser vivenciadas pelos consumidores. Sugere-se, então, que sejam incluídas experiências de produtos variando de um período curto de tempo (um dia, por exemplo) a um período consideravelmente maior (um ano, por exemplo), o que propiciaria a verificação do papel do tempo no processo.

Outra via importante de estudos está na relação entre a surpresa e os desdobramentos da satisfação, como lealdade e propaganda boca a boca. A investigação da influência que a surpresa pode ter em comportamentos futuros, mediada ou não pela satisfação, é algo pouco explorado na academia (com a exceção de Derbaix e Vanhamme [2003] que examinaram a relação entre surpresa e propaganda boca a boca) e de importante implicação gerencial, pois o impacto da surpresa pode estender-se a comportamentos futuros e não se sabe ainda se sua ação é tópica ou pode ter consequiências de longo prazo.

Seria recomendável estudar adicionalmente os antecedentes das surpresas negativa e positiva, principalmente no que diz respeito à influência da experiência passada com o produto. Produtos maduros ou inovadores, por exemplo, podem causar diferentes efeitos na satisfação e suas conseqüências, se experiências surpreendentes ocorrerem. Além disso, é importante aprender em que ambientes competitivos e em que combinações de produto-mercado a surpresa positiva pode 


\section{SURPRES A E A FORMAÇÃO DA SATISFAÇÃO DO CONSUMIDOR}

Juliano A. Larán - Carlos Alberto Vargas Rossi

proporcionar alto impacto às práticas de marketing, sendo seu estudo de elevada importância acadêmica e gerencial.

Efeitos de assimilação e contraste também podem impactar o grau de surpresa das experiências. Segundo Oliver (1997), a assimilação refere-se à relutância do indivíduo em reconhecer discrepâncias em relação a uma posição previamente tomada, enquanto o efeito de contraste é justamente o oposto, caracterizado por uma tendência para exagerar a discrepância entre as próprias atitudes e a atitude de pessoas como uma opinião diferente. Aplicado ao marketing, isso significa que o desempenho ruim de um produto/serviço será exagerado, bem como o bom desempenho será colocado em um patamar superior ao que deveria. Portanto, é importante verificar até que ponto os consumidores tendem a não relatar que ficaram de fato surpresos com determinada experiência (assimilação) ou exagerar o grau de surpresa (contraste), algo obtido a partir de um design de pesquisa que avalie esses efeitos de forma específica.

Os resultados da pesquisa trazem evidências de que um mesmo produto não terá efeito simétrico (em sentido oposto) para efeitos de surpresa negativa e positiva. É importante sublinhar que, apesar de ter sido encontrado um impacto mais forte da surpresa negativa, a assimetria não se comportará necessariamente dessa forma em todas as situações. A sugestão de pesquisa atrelada a essas considerações é de que sejam investigadas as razões subjacentes aos efeitos assimétricos da surpresa, pois o fato de eles não serem sistemáticos requer a proposição de teorias que permitam a explicação do fenômeno.

\section{Limitações do estudo}

Este estudo apresenta limitações de natureza teórica e metodológica. Uma primeira e importante limitação concerne ao inevitável caráter parcial das descobertas. Os resultados sobre a surpresa e as emoções foram alcançados por uma rota eminentemente quantitativa, o que denota o acesso a apenas uma forma de obtenção de informação sobre esses fenômenos. A amostra constituída de estudantes é outra limitação. A literatura não é conclusiva quanto ao tipo de amostra mais adequado em pesquisas da área de ciências sociais ou comportamento do consumidor. No entanto, é acertado dizer que estudantes não podem representar comportamentos de populações inteiras e que uma amostra aleatória, mais heterogênea, traria maior possibilidade de generalização dos resultados obtidos. O ideal, nesse caso, seria utilizar uma amostra de estudantes e testar os resultados em outra amostra, mais 
representativa da população, o que seria importante para a avaliação das características psicométricas das escalas utilizadas e para o teste do modelo.

A presente pesquisa, de corte transversal, operacionalizada a partir de uma survey, não permite, por outro lado, inferências de causa e efeito. Dessa forma, sugere-se a realização de experimentos com a manipulação da variável surpresa, o que tornaria essa emoção mais forte para a verificação de sua influência na satisfação.

\section{NOTA}

1 Nos estudos de marketing, os termos "afeto" e "emoção" são utilizados de forma intercambiável (veja Babin et al., 1998; Dubé e Morgan, 1998). Apesar de estarem relacionados, o termo afeto é considerado um "guarda-chuva" para uma série de processos mentais que incluem emoções, humor e atitudes (Bagozzi et al., 1999). Considerando estes estudos recentes na área de marketing e o fato de que as emoções parecem representar formas de afeto, os dois termos são utilizados neste trabalho, pois, ao tratar das emoções que determinam a satisfação, estar-se-á tratando também da afetividade positiva e negativa incutida nessas emoções.

\section{REFERÊNCIAS BIBLIOGRÁFICAS}

ARRUDA, A. L. Avaliação da aplicabilidade da escala Consumption Emotions Set para mensuração das emoções em experiências de consumo na cidade de Porto Alegre. Dissertação de Mestrado, PPGA/UFRGS, 2002.

BABIN, Barry J.; DARDEN, William R.; BABIN, Laurie A. Negative emotions in marketing: affect or artifact? Journal of Business Research, vol. 42, p. 271-285, 1998.

BAGOZZI, R. P.; GOPINATH, Mahesh; NYER, Prashanth U. The role of emotions in marketing. Journal of the Academy of Marketing Science, vol. 27, n. 2, p. 184-206, spring 1999. 
BAGOZZI, R.P.; PHILLIPS, L. W. Representing and testing organizational theories: a holistic construal, Administrative Science Quarterly, vol. 27, p. 459-489, 1992.

BEBER, S. J. N. Análise da insatisfação do consumidor em relação aos serviços prestados por assistência técnica de automóveis. Dissertação de Mestrado, PPGA/UFRGS, 2000.

BRUNER II, Gordon C.; HENSEL, Paul J. Marketing scales handbook: a compilation of multi-item measures. vol. II. Chicago: American Marketing Association, 1998.

DERBAIX, C.; PHAM, M. T. Affective reactions to consumption situations: a pilot investigation. Journal of Economic Psychology, vol. 12, p. 325-355, 1991.

DERBAIX, C.; VANHAMME, Joëlle. Inducing word-of-mouth by eliciting surprise - a pilot investigation. Journal of Economic Psychology, vol. 24, p. 99-116, 2003.

DUBÉ, Laurette; MORGAN, Michael S. Capturing the dynamics of in-process consumption emotions and satisfaction in extended service transactions. International Journal of Research in Marketing, vol. 15, p. 309-320, 1998.

FARIAS, S. A.; SANTOS, R. C. Atributos de satisfação nos serviços de hotelaria: uma perspectiva no segmento de terceira idade. In: ENCONTRO ANUAL DA ASSOCIAÇÃO NACIONAL DOS PROGRAMAS DE PÓS-GRADUAÇÃO EM ADMINISTRAÇÃO, 22., 1998, Foz do Iguaçú. Anais. Foz do Iguaçu: ANPAD, 1998.

FISCHOFF, B. Hindsight-foresight: the effect of outcome knowledge on judgment under uncertainty. Journal of Experimental Psychology: Human Perception and Performance, 1, p. 288-299, 1975.

FONSECA, M. J. Avaliação da aplicabilidade da escala New Involvement Profile para mensuração do envolvimento do consumidor na cidade de Porto Alegre. Dissertação de Mestrado - PPGA/UFRGS, 1999. 
FONSECA, M. J.; ROSSI, C. A. V. Avaliação da aplicabilidade da escala New Involvement Profile para mensuração do envolvimento do consumidor na cidade de Porto Alegre. In: ENCONTRO ANUAL DA ASSOCIAÇÃO NACIONAL DOS PROGRAMAS DE PÓS-GRADUAÇÃO EM ADMINISTRAÇÃO, 23., 1999, Foz do Iguaçú. Anais. Foz do Iguaçú: ANPAD, 1999.

FONSECA, M. J.; ROSSI, C. A. V. Involvement research in an international context: evaluation of a scale in Brazil. In: AMA's 2000 Summer Marketing Educators' Conference - AMA, 2000, Chicago, Estados Unidos. Proceedings. American Marketing Association, 2000, p. 287-296.

FORNELL, C.; LARCKER, D. F. Evaluating structural equation models with unobserved variables and measurement error. Journal of Marketing Research, v.18, p. 39-50, feb. 1981.

FRIJDA, N. The emotions. Cambridge: Cambridge University Press, 1986.

GIGLIO, E.A.; CHAUVEL, M A. Reclamação e cultura brasileira: um estudo baseado na análise de cartas de consumidores insatisfeitos à imprensa. In: ENCONTRO ANUAL DA ASSOCIAÇÃO NACIONAL DOS PROGRAMAS DE PÓS-GRADUAÇÃO EM ADMINISTRAÇÃO, 26., 2002, Salvador. Anais. Salvador: ANPAD, 2002.

HAIR, Jr. Joseph F.; ANDERSON, Rolph E.; TATHAM, Ronald L.; BLACK, William C. Multivariate data analysis. 5.ed. New Jersey: Prentice Hall, 1998.

HOLBROOK, Morris B.; HIRSCHMAN, Elizabeth C. The experiential aspects of consumption: consumer fantasies, feelings, and fun. Journal of Consumer Research, vol. 9, n. 2, p. 132-140, sep. 1982.

IZARD, Carroll E. Human emotions. New York: Plenum, 1977. 
IZARD, C. E.; BUECHLER, S. Aspects of consciousness and personality in terms of differential emotions theory. In Plutchik, R. e Kelerman (eds.). Emotion: theory, research, and experience, vol. 1, New York: Academic Press, p. 165-187, 1980.

JAGPAL, H. Multicollinearity in structural equation models with unobservable variables. Journal of Marketing Research, vol. 19, p. 431-439, nov. 1982.

JAIN, K.; SRINIVASAN, N. An empirical assessment of multiple operationalizations of involvement. In: Advances in Consumer Research, 1990, Provo, UT. Proceedings. Provo, UT: Association for Consumer Research, vol. 17, p. 594-602, 1990.

KAHNEMAN, Daniel; MILLER, Dale T. Norm theory: comparing reality to its alternatives. Psychological Review, vol. 93, n. 2, p. 136-153, 1986.

KAHNEMAN, D.; TVERSKY, A. Prospect theory: an analysis of decision under risk. Econometrica, vol. 47, p. 263-291, mar.1979.

KLINE, R. B. Principles and practice of structural equation modeling. New York: The Guilford Press, 1998.

KUMAR, A. Customer delight: creating and maintaining competitive advantage. Bloomington: Indiana University, 1996. Doctoral Dissertation (Doctor of Philosophy in Business Administration), School of Business, Indiana University, 1996.

KUMAR, A; OLSHAVSKY, R. W.; KING, M. F. Exploring alternative antecedents of customer delight. Journal of Consumer Satisfaction, Dissatisfaction and Complaining Behavior, vol. 14, p. 14$26,2001$.

LARAN, J. A. A influência da surpresa no processo emocional de formação da satisfação do consumidor. Dissertação de Mestrado - PPGA/UFRGS, 2002. 
MANO, H.; OLIVER, R. L. Assessing the dimensionality and structure of the consumption experience: evaluation, feeling and satisfaction. Journal of Consumer Research, vol. 20, p. 451-466, dec. 1993.

MARCHETTI, R. Z.; PRADO, P. Avaliação da satisfação do consumidor utilizando o PLS: um modelo aplicado ao setor elétrico brasileiro. In: ENCONTRO ANUAL DA ASSOCIAÇÃO NACIONAL DOS PROGRAMAS DE PÓS-GRADUAÇÃO EM ADMINISTRAÇÃO, 25., 2001, Campinas. Anais. Campinas: ANPAD, 2001.

MEYER, W-U; NIEPEL, M.; SCHÜTZWÖHL. An experimental analysis of surprise. Cognition and Emotion, v. 5, p. 295-311, 1991.

OATLEY, K.; JOHNSON-LAIR, P. N. Towards a cognitive theory of emotions. Cognition and Emotion, vol. 1, p. 2-50, 1987.

OLIVER, R. L. Cognitive, affective, and attribute bases of the satisfaction response. Journal of Consumer Research, vol. 20, p. 418-430, dec. 1993.

OLIVER, R. L. Processing of the satisfaction response in consumption: a suggested framework and research propositions. Journal of Consumer Satisfaction, Dissatisfaction and Complaining Behavior, vol. 2, p. 1-16, 1989.

OLIVER, R. L. Satisfaction: a behavioral perspective on the consumer. New York: McGraw Hill, 1997.

OLIVER, R. L.; RUST, R. T.; VARKI, S. Customer delight: foundations, findings, and managerial insight. Journal of Retailing, v. 73, n. 3, p. 311-336, 1997.

OLIVER, R. L.; WESTBROOK, R. A. Profiles of consumer emotions and satisfaction in ownership and usage. Journal of Consumer Satisfaction, Dissatisfaction and Complaining Behavior, vol. 6, p. 1227, 1993. 
ORTONY, A.; ClORE, G. L.; COLLINS, A. The cognitive structure of emotions. Cambridge: Cambridge University Press, 1988.

PLUTCHIK, R. Emotion: a psychoevolutionary synthesis. New York: Harper \& Row, 1980.

ROSA, F.; KAMAKURA, W. Canais de atendimento eletrônico e satisfação, retenção e rentabilidade de clientes em bancos: um estudo em nível de indivíduo. In: ENCONTRO ANUAL DA ASSOCIAÇÃO NACIONAL DOS PROGRAMAS DE PÓS-GRADUAÇÃO EM ADMINISTRAÇÃO, 26., 2002, Salvador. Anais. Salvador: ANPAD, 2002.

ROSSI, C. A. V.; HOR-MEYLL, L. F. Explorando novas trilhas na pesquisa do consumidor. In: ENCONTRO ANUAL DA ASSOCIAÇÃO NACIONAL DOS PROGRAMAS DE PÓSGRADUAÇÃO EM ADMINISTRAÇÃO, 25., 2001, Campinas. Anais. Campinas: ANPAD, 2001.

ROSSI, C. A. V.; SLONGO, L.A. Pesquisa de satisfação de clientes: o estado-da-arte e proposição de um método brasileiro. In: ENCONTRO ANUAL DA ASSOCIAÇÃO NACIONAL DOS PROGRAMAS DE PÓS-GRADUAÇÃO EM ADMINISTRAÇÃO, 21., 1997, Rio das Pedras. Anais. Rio das Pedras: ANPAD, 1997.

RUMELHART, D. E. Schemata and the cognitive system. In: Wyer, R. S. e Srull, T. K. (eds.). Handbook of social cognition, 1, Hillsdale, NJ: Lawrance Erlbaum Associates Inc., p. 161-188, 1984.

SANTOS, C. P. Impacto do gerenciamento de reclamações na confiança e lealdade do consumidor, no contexto de trocas relacionais de serviços: construção e teste de um modelo teórico. Tese de Doutorado, PPGA/UFRGS, 2001.

SPRENG, R. A.; MACKENZIE, S. B.; OLSHAVSKY, R. W. A reexamination of the determinants of consumer satisfaction. Journal of Marketing, v. 60, p. 15-32, jul. 1996.

STIENSMEIER-PELSTER, J.; MARTINI, A.; REISENZEIN. The role of surprise in the attribution process. Cognition and Emotion, v. 9, p. 5-31, 1995. 
TABACHNICK, B. G.; FIDELL, L. S. Using multivariate statistics. Needham Heights: Allyn \& Bacon, 2001.

URDAN, A. T.; RODRIGUES, A. R. O modelo do índice de satisfação do cliente norte-americano: um exame inicial no Brasil com equações estruturais. In: ENCONTRO ANUAL DA ASSOCIAÇÃO NACIONAL DOS PROGRAMAS DE PÓS-GRADUAÇÃO EM ADMINISTRAÇÃO, 22., 1998, Foz do Iguaçú. Anais. Foz do Iguaçú: ANPAD, 1998.

VANHAMME, J. Comunicação pessoal por e-mail, jan. 2003.

. La surprise et son influence sur la satisfaction des consommateurs: le cas de l'expérience de consommation/achat. Louvain-la-Neuve, Bélgica: Université Catholique de Louvain, 2002. Thèse Doctorale (Doctorat en Sciences de Gestion), Institut d'Administration et de Gestion - Unité de Marketing, Université Catholique de Louvain, 2002-a.

L'influence de la surprise sur la satisfaction des consommateurs - étude exploratoire par journal de bord. Recherche et Applications en Marketing, vol. 16, n. 2, p. 1-31, 2001.

. L'influence de la surprise sur la satisfaction des consommateurs: une expérimentation pilote. In: Conférence de l'Association Française du Marketing, 2002-b, Lille. Actes. Association Française du Marketing, 2002-b. vol. 14, p. 17-43.

. The influence of the emotion of surprise on consumer's satisfaction: a pilot experiment. In: 31st Conference of European Marketing Academy - EMAC, 2002, Braga, Portugal. Proceedings. European Marketing Academy, 2002-c, p. 0-6.

. The link between surprise and satisfaction: an exploratory study on how to best measure surprise. Journal of Marketing Management, vol. 16, p. 565-582, 2000.

VANHAMME, J.; LINDGREEN, A. Gotcha! Findings from an exploratory investigation on the dangers of using deceptive practices in the mail-order business. Psychology and Marketing, vol. 18, n. 7, p. 785-810, jul. 2001. 
VANHAMME, J.; SNELDERS, D. The role of surprise in satisfaction judgements. Journal of Consumer Satisfaction, Dissatisfaction and Complaining Behavior, vol. 14, p. 27-45, 2001.

WESTBROOK, R. A. A rating scale for measuring product/service satisfaction. Journal of Marketing, vol. 44, p. 68-72, 1980.

. Product/consumption-based affective responses and postpurchase processes. Journal of Marketing Research, vol. 24, p. 258-270, aug. 1987.

WESTBROOK, R. A.; OLIVER, R. L. The dimensionality of consumption emotion patterns and consumer satisfaction. Journal of Consumer Research, vol. 18, p. 84-91, jun. 1991.

WILliAMS, P.; AAKER, J. L. Can mixed emotions peacefully coexist? Journal of Consumer Research, vol.28, n. 4, p. 636-649, 2002.

\section{Artigo recebido em 21.07.2003. Aprovado em 08.12.2004.}

\section{Juliano A. Larán}

Doutorando no departamento de marketing da University of Florida.

Interesses de pesquisa na área de processamento de informações em comportamento de compra.

E-mail: laran@ufl.edu

Endereço: Department of Marketing - University of Florida. P.O. Box 117155, Gainesville, FL 326117155, USA.

\section{Carlos Alberto Vargas Rossi}

Professor do PPGA-UFRGS. Doutor pela FEA-USP.

Interesses de pesquisa nas áreas de comportamento do consumidor e marketing estratégico.

E-mail: cavrossi@ea.ufrgs.br

Endereço: EA - UFRGS, Washington Luiz, 855, sala 445, Centro, Porto Alegre - RS, 90010-460. 